\section{Prevalência e fatores associados a não utilização de mamografia em mulheres idosas}

\author{
Prevalence of non-utilization of mammography and \\ associated factors in elderly women
}

\begin{abstract}
This study analyzes the prevalence of non-utilization of mammography among older women, according to socio-demographic variables, health status, and use of preventive health services. This was a cross-sectional study including women 60 years or older. We interviewed 4,621 women 60 to 106 years of age; the majority were widows (51.8\%) and had little or no schooling (53.8\%). Most (89\%) reported health problems, and 66.4\% used private medical care. Prevalence of self-reported mammography was $72.1 \%$. Gynecological visits ( $P R=2.39 ; 95 \% C I$ : 2.04-2.80), Pap smear $(P R=3.24 ; 95 \% C I: 2.89-3.63)$, years of schooling $(P R=1.07 ; 95 \% C I: 1.02-1.12)$, health care insurance $(P R=1.16 ; 95 \% C I: 1.11-1.20)$, physician visits $(P R=1.23 ; 95 \% C I: 1.11-1.37)$, age $(P R=$ 1.12; 95\% CI: 1.08-1.17), marital status ( $P R=1.05$; 95\%CI: 1.00-1.09), and barriers to health services (PR $=0.94$; 95\%CI: 0.89-0.98) were also associated with non-utilization of mammography. These associations may be partially explained by lack of knowledge, poor access to public health services, and cultural factors related to the aging process and reproductive incapacity.
\end{abstract}

Mammography; Aging; Breast Neoplasms; Mass Screening
Cristiane de Oliveira Novaes 1

Inês Echenique Mattos 1

\section{Introdução}

A análise da mortalidade entre os idosos brasileiros, no período de 1980-2000 1, mostra a importância das neoplasias como causa de morte neste grupo populacional e os tumores de mama como a principal localização de câncer nas mulheres.

O diagnóstico da neoplasia de mama em estágios iniciais pode reduzir a mortalidade, aumentando a probabilidade de sobrevida das mulheres acometidas $2 \mathrm{e}$, neste sentido, a mamografia, como método diagnóstico, é a estratégia mais efetiva, mesmo para esse grupo etário 3,4. O rastreamento através da mamografia é capaz de detectar a doença ainda em fase inicial, o que pode melhorar o prognóstico 5 . Estudos recentes indicam que o rastreamento regular do câncer de mama em mulheres com 50 ou mais anos de idade pode reduzir a mortalidade em até $45 \%$ 6,7.

Entretanto, ainda não existe um consenso internacional que estabeleça critérios claros no caso de mulheres em idade avançada. Até o final da década de 90, alguns grupos recomendavam a cessação do rastreamento aos 70 anos ${ }^{8}$. Em anos mais recentes, diferentes autores passaram a recomendar a realização de rastreamento mamográfico para mulheres idosas 9,10. A American Cancer Society recomenda que, devido à heterogeneidade da população idosa, a idade cronológica não seja utilizada como critério isolado para a cessação desse exame, devendo ser também considerados outros fatores como expecta- 
tiva de vida, presença de doenças e capacidade funcional 11 .

No Brasil, o Consenso para o Controle de Câncer da Mama preconiza a realização do exame clínico das mamas para mulheres em todas as faixas etárias, como parte do atendimento integral à mulher e, em relação à mamografia, recomenda sua realização a cada dois anos, para mulheres de 50 a 69 anos 12 .

Diversos estudos 13,14,15,16,17 que analisaram fatores relacionados à utilização da mamografia evidenciaram a associação entre idade, escolaridade, nível sócio-econômico, estado conjugal, história familiar de câncer e recomendação médica, e a adesão ao rastreamento. Contudo, o conhecimento da prevalência de realização de mamografia na parcela idosa da população brasileira, bem como dos fatores individuais relacionados à utilização ou não deste exame em idades mais avançadas, são restritos às mulheres até 69 anos, ou resultam de inquéritos gerais de saúde que estimaram esta medida, mas não exploraram as características associadas à adesão $3,18,19,20,21,22,23$.

O limite etário presente nos estudos sobre essa temática se contrapõe às recomendações que apontam a idade cronológica como critério insuficiente para estabelecer a população-alvo do rastreamento. Ainda existe, portanto, uma lacuna, seja na literatura nacional, ou internacional, no que tange ao conhecimento da prevalência da não utilização de mamografia e dos fatores a ela associados, na população feminina de 70 ou mais anos de idade.

Face ao exposto, o objetivo deste estudo foi estimar a prevalência de realização de mamografia em mulheres com 60 ou mais anos, residentes em Juiz de Fora, Minas Gerais, Brasil, que participaram da campanha de vacinação contra a gripe de 2006, e analisar os fatores associados a não utilização desse exame em um grupo populacional que, de alguma forma, utiliza o serviço público de saúde.

\section{Material e métodos}

O estudo EPOCA - Envelhecimento Populacional $e$ Câncer foi realizado durante o período oficial da campanha nacional de vacinação contra gripe de 2006, sendo entrevistados 7.531 indivíduos idosos de ambos os sexos. Os critérios de exclusão considerados foram: idade inferior a 60 anos, incapacidade física e/ou cognitiva severa, residência em outro município ou em área rural e vacinação realizada em postos móveis no dia nacional de vacinação contra gripe. O questionário do estudo foi construído com base em instrumentos padro- nizados e validados para a população brasileira, que já haviam sido utilizados em outros estudos nacionais 20,22,23.

Durante a campanha, foram organizados 41 postos de vacinação na zona urbana de Juiz de Fora, distribuídos nas cinco regiões geográficas da cidade, pelo período de 11 dias, que funcionavam das 8 às 17 horas, exceto aos domingos. A adesão do idoso à vacinação era voluntária, gratuita e independente da área de moradia, sendo que a divulgação ocorreu nos principais meios de comunicação locais, regionais e nacionais, além de ser estimulada por parte dos profissionais das unidades de saúde.

A estratégia de captar a população de estudo durante a campanha foi adotada com o objetivo de alcançar um conjunto de mulheres que abrangesse, inclusive, aquelas que se dirigem às unidades de saúde da rede pública apenas na campanha de vacinação, por terem como referência, para uso geral, os serviços privados. As mulheres foram abordadas nos postos de vacinação, quando foram explicados os objetivos do estudo, e aquelas que aceitaram participar assinaram o termo de consentimento informado e responderam a um questionário estruturado, aplicado por entrevistadores treinados.

A logística do estudo incluiu o envio simultâneo de entrevistadores a todos os postos de vacinação, durante os 11 dias de campanha, nos dois turnos de funcionamento de cada posto. Cabe ressaltar que os entrevistadores foram alocados em função da relação quantidade/demanda esperada, tendo como base dados de campanhas anteriores. Foram empreendidos esforços para atingir o maior número possível de idosos.

Em 2006, a cobertura atingiu $73 \%$ da população-alvo da campanha, sendo aplicadas 39.522 doses de vacina. O cálculo da cobertura e das doses inclui a vacinação em domicílio de idosos acamados, indivíduos residentes na área rural, pacientes de risco vacinados independente da idade, indivíduos fora da faixa etária ou residentes em outras cidades, além de pessoas que receberam a vacina em um período de duas semanas após a campanha. Não existem dados que possibilitem identificar a proporção de vacinados por sexo ou faixa etária.

Esta investigação consiste em um estudo transversal com mulheres residentes em Juiz de Fora, com idade igual ou superior a 60 anos, que compareceram à campanha. A variável desfecho do estudo foi "não realização de mamografia nos últimos dois anos" e as variáveis independentes consideradas foram: características sócio-demográficas - idade, situação conjugal (agrupado em ter ou não cônjuge, com as solteiras, viúvas e separadas integrando a categoria sem cônju- 
ge), escolaridade e renda (estratificada em dois grupos, sendo o parâmetro ter renda declarada acima ou até três salários mínimos, considerando o valor vigente em abril de 2006 , de $\mathrm{R} \$ 350,00$ ); características relacionadas ao estado de saúde - uso regular de medicação, doenças auto-referidas, história familiar de câncer de útero, história familiar de câncer de mama e auto-avaliação da saúde, por meio da pergunta "Como é a sua saúde de modo geral: excelente, boa, razoável ou ruim?”; características das práticas preventivas e de cuidados à saúde - serviço de saúde de referência, visitas ao médico, ocorrência de internação no último ano, realização de histerectomia e perfil de utilização dos exames preventivos nos últimos dois anos (Papanicolaou, mamografia e exame clínico das mamas feito por médico).

Foram realizadas análises univariadas para a descrição das características sócio-demográficas, do estado de saúde e das práticas preventivas e de cuidados à saúde. As análises bivariadas estimaram as prevalências e, posteriormente, as razões de prevalência da não utilização de mamografia, com o intervalo de $95 \%$ de confiança (IC95\%), explorando o tempo transcorrido desde o último exame, bem como o motivo da sua realização. Além disso, foram estimadas as médias, medianas e variâncias quando pertinentes.

Para a análise multivariada, consideraram-se as variáveis que apresentaram valor de $p<0,20$ na análise bivariada. A análise foi ajustada por meio da regressão de Poisson, com a inclusão de uma variável de cada vez no modelo, tendo permanecido apenas aquelas que apresentaram valor de $\mathrm{p}<0,05$. Foram utilizados os programas SPSS 11.0 (SPSS Inc., Chicago, Estados Unidos) e Stata 7.0 (Stata Corp., College Station, Estados Unidos). O projeto foi aprovado pelo Comitê de Ética em Pesquisa da Escola Nacional de Saúde Pública Sergio Arouca, Fundação Oswaldo Cruz.

\section{Resultados}

Foram entrevistadas 4.621 mulheres, com idades entre 60 e 106 anos (média de idade: 70,03; desvio-padrão- $\mathrm{DP}=7,14$ ), sendo a maior proporção constituída pela faixa etária 60 a 69 anos. A maior parcela era formada por viúvas $(51,8 \%)$, com até quatro anos de estudos (53,8\%), renda de até três salários mínimos $(80,2 \%)$ e usuárias $(66,4 \%)$ dos serviços públicos de saúde (Tabela 1 ).

Quanto às características relacionadas ao estado de saúde, a maioria das entrevistadas avaliou a própria saúde física como boa ou regular, $83,7 \%$ utilizavam regularmente pelo menos um medicamento e $89 \%$ referiram ter pelo menos uma doença (média: 2,39; DP = 1,78). A minoria
(15,8\%) relatou internação nos 12 meses anteriores à entrevista e 20,8\% haviam sido submetidas à histerectomia. História familiar de câncer de mama ou de útero foi relatada por 14,5\% e 8,7\% das mulheres, respectivamente (Tabela 1).

Em relação às práticas preventivas e ao autocuidado, a maior parte $(95,6 \%)$ relatou ter feito pelo menos uma consulta médica no último ano, e cerca de $70 \%$ possuíam um médico especialista de referência. Quanto à consulta ginecológica, $91,1 \%$ referiram ter feito pelo menos uma em toda a vida. Foi observado alto porcentual de realização de exame de Papanicolaou (86\%), sendo que entre as mulheres que haviam feito este exame pelo menos uma vez na vida, 96,6\% informaram como motivo o rastreamento para câncer de colo uterino e 76,3\% relataram que o tinham realizado nos últimos dois anos. Quanto ao exame clínico de mamas, $80,9 \%$ relataram terem sido examinadas pelo menos uma vez na vida, sendo o rastreamento para câncer de mama o principal motivo alegado (97,6\%), e 79,7\% referiram o exame nos últimos dois anos (Tabela 2).

A mamografia foi realizada pelo menos uma vez na vida por $72,1 \%$ das entrevistadas, sendo que praticamente todos os exames realizados $(98,8 \%)$ tiveram a finalidade de rastreamento e foram efetuados nos últimos dois anos $(92,6 \%)$. Das mulheres que realizaram o exame nos últimos dois anos, $53,7 \%$ o fizeram no sistema público. A idade mínima quando da realização do último exame foi de 57 anos e a máxima de 94 , com média de 67,63 anos (DP =6,50) e mediana de 66 anos.

Na Tabela 3, verifica-se que a prevalência de não realização de mamografia se mostrou associada a variáveis sócio-demográficas, do estado de saúde e de práticas preventivas. Entre as variáveis demográficas, ser mais velha (razão de prevalência - RP = 1,25; IC95\%: 1,20-1,29), viver sem companheiro (RP = 1,18; IC95\%: 1,14-1,22), ter pouca escolaridade $(\mathrm{RP}=1,24$ : IC95\%: 1,20 $1,29)$ e renda abaixo de três salários mínimos $(\mathrm{RP}=1,23$; IC95\%: 1,19-1,27) foram fatores de risco para a não realização da mamografia. Em relação às variáveis do estado de saúde e das práticas preventivas, os fatores de risco associados foram: ser dependente do Sistema Único de Saúde-SUS (RP = 1,26; IC95\%: 1,21-1,30), ter uma auto-avaliação negativa da saúde $(\mathrm{RP}=1,05$; IC95\%: 1,011,09), não ter realizado consulta médica no último ano (RP = 1,55; IC95\%: 1,34-1,79), não ter consultado um ginecologista $(\mathrm{RP}=6,12$; IC95\%: 4,75-7,90), não ter feito o exame de Papanicolaou ( $R P=5,09$; IC95\%: 4,27-6,08) ou exame clínico das mamas $(\mathrm{RP}=21,01$; IC95\%: 15,32-28,80). Como fatores protetores, foram identificados: ter história familiar de câncer de mama $(\mathrm{RP}=0,90$; IC95\%: 
Tabela 1

Estudo EPOCA - Envelhecimento Populacional e Câncer: características sócio-demográficas e de estado de saúde da população de estudo. Juiz de Fora, Minas Gerais, Brasil, 2006

\begin{tabular}{|c|c|c|}
\hline Características & $\mathrm{n}$ & $\%$ \\
\hline \multicolumn{3}{|l|}{ Sócio-demográficas } \\
\hline \multicolumn{3}{|l|}{ Idade (anos) } \\
\hline $60-69$ & 2.432 & 52,6 \\
\hline $70-79$ & 1.661 & 35,9 \\
\hline $80-89$ & 499 & 10,8 \\
\hline 90 ou mais & 29 & 0,6 \\
\hline \multicolumn{3}{|l|}{ Estado conjugal } \\
\hline Solteira & 510 & 11,1 \\
\hline Casada & 1.713 & 37,1 \\
\hline Viúva & 2.392 & 51,8 \\
\hline \multicolumn{3}{|l|}{ Escolaridade } \\
\hline Menos de 4 anos & 2.487 & 53,8 \\
\hline 4 anos completos & 1.317 & 28,5 \\
\hline Entre 5 e 11 anos & 574 & 12,4 \\
\hline Superior & 243 & 5,3 \\
\hline \multicolumn{3}{|c|}{ Renda (salários mínimos) } \\
\hline Até 3 & 3.708 & 80,2 \\
\hline $3-8$ & 636 & 13,8 \\
\hline Acima de 8 & 277 & 6,0 \\
\hline \multicolumn{3}{|c|}{ Tipo de serviço de saúde que utiliza } \\
\hline Público & 3.069 & 66,4 \\
\hline Privado & 1.552 & 33,6 \\
\hline \multicolumn{3}{|l|}{ Estado de saúde } \\
\hline \multicolumn{3}{|c|}{ Auto-avaliação da saúde } \\
\hline Ruim & 299 & 6,5 \\
\hline Regular & 1.829 & 39,6 \\
\hline Boa & 2.007 & 43,4 \\
\hline Excelente & 486 & 10,5 \\
\hline \multicolumn{3}{|l|}{ Uso de medicação } \\
\hline Sim & 3.869 & 83,7 \\
\hline Não & 752 & 16,3 \\
\hline \multicolumn{3}{|c|}{ Doença crônica auto-referida } \\
\hline Sim & 4.113 & 89,0 \\
\hline Não & 508 & 11,0 \\
\hline \multicolumn{3}{|c|}{ Relato de internação no último ano } \\
\hline Sim & 729 & 15,8 \\
\hline Não & 3.892 & 84,2 \\
\hline \multicolumn{3}{|l|}{ Histerectomia } \\
\hline Sim & 961 & 20,8 \\
\hline Não & 3.660 & 79,2 \\
\hline \multicolumn{3}{|c|}{ História familiar de câncer de mama } \\
\hline Sim & 668 & 14,5 \\
\hline Não & 3.953 & 85,5 \\
\hline \multicolumn{3}{|c|}{ História familiar de câncer de útero } \\
\hline Sim & 401 & 8,7 \\
\hline Não & 4.220 & 91,3 \\
\hline
\end{tabular}


Estudo EPOCA - Envelhecimento Populacional e Câncer: características relacionadas às práticas preventivas e de cuidado à saúde. Juiz de Fora, Minas Gerais, Brasil, 2006.

\begin{tabular}{|c|c|c|}
\hline Características & $\mathrm{n}$ & $\%$ \\
\hline \multicolumn{3}{|c|}{ Relato de consulta médica no último ano } \\
\hline Sim & 4.418 & 95,6 \\
\hline Não & 203 & 4,4 \\
\hline \multicolumn{3}{|c|}{ Possui algum médico especialista de referência } \\
\hline Sim & 3.269 & 70,7 \\
\hline Não & 1.352 & 29,3 \\
\hline \multicolumn{3}{|c|}{ Relato de consulta com ginecologista } \\
\hline Sim & 4.212 & 91,1 \\
\hline Não & 409 & 8,9 \\
\hline \multicolumn{3}{|c|}{ Realização de exame de Papanicolaou } \\
\hline Sim & 3.975 & 86,0 \\
\hline Não & 646 & 14,0 \\
\hline \multicolumn{3}{|c|}{ Motivo do exame de Papanicolaou } \\
\hline Diagnóstico & 85 & 2,1 \\
\hline Rastreamento & 3.842 & 96,6 \\
\hline \multicolumn{3}{|c|}{ Tempo do último exame de Papanicolaou (anos) } \\
\hline Até 2 & 3.035 & 76,3 \\
\hline$>2$ & 564 & 14,2 \\
\hline \multicolumn{3}{|c|}{ Realização de exame clínico das mamas } \\
\hline Sim & 3.739 & 80,9 \\
\hline Não & 882 & 19,1 \\
\hline \multicolumn{3}{|c|}{ Motivo do exame clínico das mamas } \\
\hline Diagnóstico & 51 & 1,4 \\
\hline Rastreamento & 3.644 & 97,6 \\
\hline \multicolumn{3}{|c|}{ Tempo do último exame clínico das mamas (anos) } \\
\hline Até 2 & 2.981 & 79,7 \\
\hline$>2$ & 420 & 11,2 \\
\hline \multicolumn{3}{|c|}{ Realização de mamografia } \\
\hline Sim & 3.332 & 72,1 \\
\hline Não & 567 & 27,9 \\
\hline \multicolumn{3}{|c|}{ Motivo da mamografia } \\
\hline Diagnóstico & 41 & 1,2 \\
\hline Rastreamento & 3.256 & 97,7 \\
\hline \multicolumn{3}{|c|}{ Tempo da última mamografia (anos) } \\
\hline Até 2 & 4.277 & 92,6 \\
\hline$>2$ & 344 & 7,4 \\
\hline
\end{tabular}

0,86-0,94), não relatar dificuldade de acesso aos serviços de saúde (RP = 0,87; IC95\%: 0,83-0,92) e a auto-referência à presença de alguma doença (RP = 0,94; IC95\%: 0,89-1,01), esta última sem significância estatística.

Após ajustamento pelas demais variáveis independentes, os fatores que permaneceram diretamente associados a não realização de mamografia foram: ausência de exame de Papanicolaou (RP = 3,24; IC95\%: 2,90-3,64), ausência de consultas com o ginecologista (RP $=2,40$; IC95\%: 2,052,81 ), ausência de consultas médicas no último ano (RP = 1,24; IC95\%: 1,12-1,37), dependência do sistema público de saúde $(\mathrm{RP}=1,16$; IC95\%: $1,12-1,20)$, idade avançada ( $R P=1,13$; IC95\%: $1,09-1,17)$, baixa escolaridade ( $\mathrm{RP}=1,07$; IC95\%: $1,02-1,12)$ e não ter um companheiro $(\mathrm{RP}=1,06$; IC95\%: 1,02-1,09). Não declarar dificuldades de acesso ao serviço de saúde $(\mathrm{RP}=0,94$; IC95\%: $0,89-0,98)$ se mostrou inversamente associado a não utilização do exame (Tabela 4). A variável não realização de exame clínico das mamas não foi testada no modelo, pois foi detectada alta correlação entre ela e o desfecho de interesse. 
Estudo EPOCA - Envelhecimento Populacional e Câncer: fatores associados a não utilização de mamografia. Juiz de Fora, Minas Gerais, Brasil, 2006.

\begin{tabular}{|c|c|c|c|}
\hline Fatores associados & $\mathrm{n}$ & $\begin{array}{c}\text { Prevalência de } \\
\text { não utilização de } \\
\text { mamografia }\end{array}$ & RP (IC95\%) \\
\hline \multicolumn{4}{|l|}{ Idade (anos) } \\
\hline $60-69$ & 2.432 & 0,20 & 1,00 \\
\hline 70 ou mais & 2.189 & 0,36 & $1,25(1,20-1,29)$ \\
\hline \multicolumn{4}{|l|}{ Estado conjugal } \\
\hline Com companheiro & 1.719 & 0,20 & 1,00 \\
\hline Sem companheiro & 2.902 & 0,32 & $1,18(1,14-1,22)$ \\
\hline \multicolumn{4}{|l|}{ Escolaridade } \\
\hline Acima de 4 anos & 817 & 0,14 & 1,00 \\
\hline Até 4 anos completos & 3.804 & 0,31 & $1,24(1,20-1,29)$ \\
\hline \multicolumn{4}{|c|}{ Renda individual mensal (salários mínimos) } \\
\hline Acima de 3 & 1.422 & 0,20 & 1,00 \\
\hline Até 3 & 3.199 & 0,31 & $1,23(1,19-1,27)$ \\
\hline \multicolumn{4}{|c|}{ Tipo de serviço de saúde que utiliza } \\
\hline Privado & 1.552 & 0,17 & 1,00 \\
\hline Público & 3.069 & 0,34 & $1,26(1,21-1,30)$ \\
\hline \multicolumn{4}{|l|}{ Auto-avaliação da saúde } \\
\hline Positiva & 2.493 & 0,26 & 1,00 \\
\hline Negativa & 2.128 & 0,30 & $1,05(1,01-1,09)$ \\
\hline \multicolumn{4}{|c|}{ Doença crônica auto-referida } \\
\hline Não & 4.113 & 0,27 & 1,00 \\
\hline Sim & 508 & 0,31 & $0,94(0,89-1,01)$ \\
\hline \multicolumn{4}{|c|}{ História familiar de câncer de mama } \\
\hline Sim & 668 & 0,21 & 1,00 \\
\hline Não & 3.953 & 0,29 & $0,90(0,86-0,94)$ \\
\hline \multicolumn{4}{|c|}{ Relato de consultas médicas no último ano } \\
\hline Sim & 4.418 & 0,27 & 1,00 \\
\hline Não & 203 & 0,53 & $1,55(1,34-1,79)$ \\
\hline \multicolumn{4}{|c|}{ Relato de consulta com ginecologista } \\
\hline Sim & 4.212 & 0,22 & 1,00 \\
\hline Não & 409 & 0,87 & $6,12(4,75-7,90)$ \\
\hline \multicolumn{4}{|c|}{ Realização de exame de Papanicolaou } \\
\hline Sim & 3.975 & 0,19 & 1,00 \\
\hline Não & 646 & 0,87 & $5,09(4,27-6,08)$ \\
\hline \multicolumn{4}{|c|}{ Realização de exame clínico das mamas } \\
\hline Sim & 3.739 & 0,12 & 1,00 \\
\hline Não & 882 & 0,96 & $21,01(15,32-28,80)$ \\
\hline \multicolumn{4}{|c|}{ Relato de dificuldades de acesso ao sistema de saúde } \\
\hline Não & 3.788 & 0,26 & 1,00 \\
\hline Sim & 833 & 0,35 & $0,87(0,83-0,92)$ \\
\hline
\end{tabular}


Estudo EPOCA - Envelhecimento Populacional e Câncer: modelo de regressão múltipla de Poisson para a não utilização de mamografia. Juiz de Fora, Minas Gerais, Brasil, 2006.

\section{$\operatorname{RP}(\mathrm{IC} 95 \%)$ *}

\begin{tabular}{|c|c|}
\hline \multicolumn{2}{|c|}{ Relato de consultas com ginecologista } \\
\hline Não & $2,39(2,04-2,80)$ \\
\hline \multicolumn{2}{|c|}{ Realização de exame de Papanicolaou } \\
\hline Não & $3,24(2,89-3,63)$ \\
\hline \multicolumn{2}{|c|}{ Relato de consultas médicas no último ano } \\
\hline Não & $1,23(1,11-1,37)$ \\
\hline \multicolumn{2}{|c|}{ Tipo de serviço de saúde que utiliza } \\
\hline Público & $1,16(1,11-1,20)$ \\
\hline \multicolumn{2}{|l|}{ Idade (anos) } \\
\hline 70 ou mais & $1,12(1,08-1,17)$ \\
\hline \multicolumn{2}{|l|}{ Escolaridade } \\
\hline Até 4 anos completos & $1,07(1,02-1,12)$ \\
\hline \multicolumn{2}{|l|}{ Estado conjugal } \\
\hline Sem companheiro & $1,05(1,01-1,09)$ \\
\hline \multicolumn{2}{|c|}{ Relato de dificuldades no acesso ao sistema de saúde } \\
\hline Não & $0,94(0,89-0,98)$ \\
\hline
\end{tabular}

* Ajustadas pelas demais variáveis do modelo.

\section{Discussão}

Estudos realizados nos Estados Unidos, que avaliaram a prevalência de utilização de mamografia em mulheres a partir de 50 anos, mostraram resultados variados. O Behavioral Risk Factor Surveillance System (BRFSS) 24 e o National Health Interview Survey (NHIS) 25 encontraram prevalência de utilização de mamografia entre $70 \%$ e $80 \%$, na população com idade igual ou superior a 65 anos. Em uma amostra de 1.111 mulheres com idades entre 50 e 75 anos, foi encontrada a prevalência de mamografia de $75 \% 16$ e, com base nos dados do Surveillance Epidemiology and End Results (SEER) 26, em uma amostra de 146.669 mulheres com idade maior ou igual a 65 anos, foi encontrada prevalência de 47,9\%. Dados do Health and Retirement Study (HRS), com amostra de 5.942 mulheres entre 50 a 61 anos, e do Asset and Health Dynamics Among the Oldest Old (AHEAD), com amostra de 4.543 mulheres com 70 ou mais anos, mostraram prevalências de $71 \%$ a $78 \%$ na faixa de 60 a 64 anos; $65 \%$ a $77 \%$ entre 70 e $74 ; 59 \%$ a $65 \%$ entre 75 e $79 ; 43 \%$ a $54 \%$ na faixa de 80 a 84 anos e $37 \%$ a $42 \%$ para mulheres com idades entre 85 e 89 anos 27. Em síntese, esses estudos evidenciam uma diminuição da prevalência de realização do exame com o aumento da idade.
No Brasil, os estudos apresentam prevalências divergentes, mas também se observa a diminuição da utilização, à medida em que a faixa etária aumenta. O Inquérito Domiciliar sobre Comportamentos de Risco e Morbidade Referida de Doenças Não-transmissíveis analisou a utilização de mamografia em mulheres com idades entre 50 e 69 anos, em 15 capitais e no Distrito Federal 20. As capitais da Região Norte apresentaram as menores prevalências de utilização de mamografia - 42,3\% para Manaus (Amazonas) e 36,8\% para Belém (Pará). Em Fortaleza (Ceará), Natal (Rio Grande do Norte), Aracaju (Sergipe), Distrito Federal e Rio de Janeiro, as prevalências observadas não atingiram $50 \%$. As maiores prevalências de utilização de mamografia foram observadas em Vitória (Espírito Santo; 76,5\%), Porto Alegre (Rio Grande do Sul; 69,9\%) e Belo Horizonte (Minas Gerais; 67,4\%). Dados da Pesquisa Nacional por Amostra de Domicílios (PNAD) mostram que, em 2003, 54\% das mulheres na faixa etária de 60 a 69 anos declararam nunca terem feito mamografia; para as que fizeram alguma vez na vida, 36,9\% haviam feito no período de dois anos ou menos e $9,1 \%$ no período de três anos ou mais ${ }^{3}$. Na PNAD, a prevalência de utilização de mamografia nos últimos dois anos decresce a partir dos 39 anos, sendo de $10,6 \%$ para o grupo de 60 a 69 anos, de $5 \%$ no grupo de 70 a 79 anos e de $1,2 \%$ pa- 
ra mulheres com 80 ou mais anos 18. A Pesquisa Mundial de Saúde no Brasil, para o ano de 2003, encontrou prevalência de utilização de mamografia de $48,6 \%$ em mulheres com idades entre 50 e 69 anos 21 .

Em Belo Horizonte, a prevalência de utilização de mamografia foi de $65 \%$ na faixa etária entre 60 e 69 anos, e correspondeu a $78,6 \%$ na faixa de 50 a 59 anos 23. Estudo de base populacional em Pelotas (Rio Grande do Sul), com uma amostra de 879 mulheres com idades entre 40 e 69 anos, encontrou que $83,7 \%$ das entrevistadas haviam feito mamografia, em um intervalo de no máximo dois anos 28. Em sete cidades da América Latina e Caribe, as prevalências variaram entre 8,2\% (Havana, Cuba) e 30,2\% (São Paulo) para mulheres com 65 anos ou mais ${ }^{19}$. Um estudo transversal de base populacional, com $1.026 \mathrm{mu}$ lheres de 20 a 60 anos residentes em São Leopoldo (Rio Grande do Sul), avaliou a desigualdade na realização do exame clínico de mama e mostrou que apenas $54,2 \%$ da população analisada havia sido submetida a este exame, observando uma associação entre classe econômica e não-realização do mesmo 29. Em um modelo restrito, que considerou somente aquelas que declararam ter feito o exame, superadas as diferenças de acesso, as mulheres brancas tiveram maior probabilidade de serem examinadas 29 .

A prevalência do uso de mamografia $(72,1 \%)$ observada no grupo avaliado mostrou-se elevada, similar à maior parte dos estudos americanos e próxima das prevalências descritas para Vitória, Porto Alegre, Belo Horizonte e Pelotas. Dados derivados da Pesquisa Mundial de Saúde 21 mostraram que a prevalência de mamografia aumenta em função do tamanho do município, superando $60 \%$ nos de médio porte (400 mil habitantes ou mais). Essa é uma possível explicação para os valores observados em Juiz de Fora, somada ao fato de que esta cidade foi pioneira em uma série de ações destinadas à população idosa, seja na área social educacional ou de saúde, tendo, inclusive, tradição em realização de campanhas de esclarecimento. A cidade também conta com uma rede de atenção distribuída pelas suas diferentes áreas. Entretanto, é importante destacar as dificuldades para comparação das prevalências de mamografia, já que os estudos nacionais, em geral, tendem a estabelecer um limite superior de 69 anos de idade, enquanto em nosso estudo foi incluída uma faixa etária mais abrangente. Além disso, embora sejam estudos relativamente recentes, os distintos períodos para a coleta dos dados poderiam também implicar em diferenças nos resultados obtidos.

Embora a prevalência de mamografia seja elevada nessa população, existe ainda um grande número de mulheres que não fazem o exame. A mamografia foi a prática preventiva que apresentou menor proporção de utilização nesse grupo, comparando-se com consulta ao ginecologista, realização de Papanicolaou e exame clínico das mamas. Além disso, é possível que, de uma maneira geral, essa parcela da população esteja também excluída de outros procedimentos, como consulta médica regular, uso de medicação e outras práticas preventivas e de autocuidado.

A característica mais fortemente associada a não utilização de mamografia foi não ter realizado o exame de Papanicolaou, seguida da não realização de consultas com ginecologista. Isso sugere a ausência de autocuidado com o aparelho reprodutor, que pode ser atribuída à perda desta função, derivada do envelhecimento. A ausência de consultas médicas e o uso de serviços públicos de saúde podem indicar dificuldade de acesso e/ou falta de autocuidado com a saúde de modo geral. Essa hipótese é reforçada, se considerarmos que não relatar dificuldades de acesso foi fator de proteção para a não realização do exame.

A idade avançada (igual ou superior a 70 anos), a baixa escolaridade e a ausência de relação conjugal mostraram-se fatores de risco para a não utilização de mamografia. As associações observadas podem ser parcialmente explicadas por fatores culturais e crenças socialmente ligadas ao envelhecimento, como as que relacionam o autocuidado do aparelho reprodutivo à existência de uma relação conjugal, à capacidade reprodutiva ou à manutenção de vida sexual ativa 30 . Outro possível fator contribuinte poderia ser a existência de patologias comuns no envelhecimento, que se tornam prioritárias para a atenção, tanto sob a ótica do indivíduo idoso quanto perante o serviço de saúde 31 , em detrimento de outros aspectos, como os exames para detecção precoce do câncer. Por fim, a falta de conhecimento sobre os riscos e possibilidades de enfrentamento do câncer de mama pode também ser um obstáculo para a busca desse exame.

Nossos achados foram similares aos observados em outros estudos nacionais e internacionais. Em uma amostra de mil mulheres idosas residentes em dois centros urbanos americanos foi observada associação positiva entre realização de rastreamento para câncer de mama e escolaridade, percepção de necessidade e cobertura para os exames $\left(\chi^{2}=29,65\right.$; valor de $\left.p<0,001\right) 32$. Outro estudo em amostra de mulheres idosas americanas 33 mostrou associação positiva entre utilização de mamografia e as variáveis nível sócio-econômico e co-habitação, além de associação negativa com idade. Já o estudo conduzido com uma amostra de 10.485 mulheres mostrou 
associação positiva entre utilização e nível sócioeconômico, escolaridade, co-habitação e número de consultas médicas, e associação negativa entre utilização e idade 27 .

Dados relacionados, derivados do inquérito sobre saúde e bem-estar de pessoas idosas residentes em sete centros urbanos da América Latina e Caribe, evidenciaram associação positiva com escolaridade e viver maritalmente 19 . Um estudo realizado em Pelotas evidenciou que quanto mais baixo o nível sócio-econômico da população, menor a proporção de mulheres idosas submetidas ao exame clínico de mamas 34 . Embora trabalhando com faixa etária distinta, que abrangia mulheres de 25 anos ou mais, a PNAD mostrou que escolaridade, renda, auto-avaliação positiva da saúde, plano de saúde, consulta médica recente e regular foram fatores positivamente associados à utilização de mamografia 18 .

Uma limitação de nosso estudo é o fato de que o uso de mamografia foi obtido por meio de auto-relato, o que poderia superestimar o uso real, conforme apontado em outros estudos 35,36. Todavia, nossos achados se mostraram consistentes com os de outros estudos nacionais e internacionais. Outra limitação consiste no fato de que os efeitos culturais, bem como os conhecimentos, crenças e atitudes sobre o rastreamento de câncer de mama, não foram examinados de forma mais aprofundada neste estudo, o que poderia ter lançado nova luz nas investigações sobre essa temática.

$\mathrm{O}$ fato de termos trabalhado com mulheres que aderiram à campanha nacional de vacinação contra a gripe, poderia ser uma limitação para a generalização dos achados para a população feminina idosa de Juiz de Fora. Entretanto, o objetivo do estudo foi investigar uma população de usuárias dos serviços de atenção, com acesso a informações de saúde (no caso a campanha) e que se reconhecem como idosas. De qualquer forma, as características gerais de saúde e o perfil sócio-demográfico das mulheres que constituíram a nossa população de estudo apresentaramse similares às de outras investigações realizadas com populações idosas brasileiras ,22,37,38,39,40,41.

A política de saúde que estabelece um limite superior de idade para uma prática de detecção precoce de câncer, como a mamografia, carrega em si uma evidência clara de discriminação etária 42 . Estudos que abordem fatores associados a não realização desse exame, e que incluam a observação de mulheres com idade superior aos 69 anos, podem contribuir para que a possibilidade de ampliar a indicação clínica seja avaliada, propiciando a adesão das mulheres que atendam aos critérios de capacidade funcional e expectativa de vida saudável. Podem também estimular a organização dos serviços de saúde e a formulação de políticas públicas que motivem a inclusão e o atendimento de uma parcela da população que permanece excluída.

Em síntese, a não realização de mamografia se mostrou associada ao pior nível sócio-econômico e a não utilização (ou utilização em menor proporção) dos serviços de saúde, de modo geral. Isso é particularmente relevante, se considerarmos que a maior parte dos idosos brasileiros se enquadra nas classes socialmente menos privilegiadas e com pior acesso à saúde 38,42. Esses achados podem refletir a iniqüidade no acesso, devido ao menor poder aquisitivo ou à crença de que não há o que ser feito, em termos de prevenção ou reabilitação em saúde, por serem pessoas idosas e estarem vivenciando um processo de declínio.

O perfil identificado sugere a necessidade de políticas mais efetivas de prevenção do câncer de mama nessa população, considerando suas especificidades, com vistas a atuar sobre os fatores que predispõe ou limitam o uso da mamografia, mas que também viabilizem o acesso aos serviços preventivos de modo geral. 


\section{Resumo}

O objetivo deste estudo transversal foi avaliar a prevalência de não utilização de mamografia em mulheres com 60 ou mais anos, de acordo com características sócio-demográficas, estado de saúde e o uso de serviços preventivos de saúde. Foram entrevistadas 4.621 mulheres, com idades entre 60 e 106 anos, a maioria viúva (51,8\%), com pouco/nenhum estudo (53,8\%), com morbidade auto-referida (89\%) e uso de serviços privados de saúde $(66,4 \%)$. A prevalência de mamografia auto-referida foi de $72,1 \%$. Consulta com ginecologista $(R P=2,39$; IC95\%: 2,04-2,80), exame de Papanicolaou $(R P=3,24$; IC95\%: 2,89-3,63), escolaridade ( $R P=1,07$; IC95\%: 1,02-1,12), serviço público de saúde (RP = 1,16; IC95\%: 1,11-1,20), consultas médicas ( $R P=1,23$; IC95\%: 1,11-1,37), idade $(R P=1,12$; IC95\%: 1,08-1,17), estado conjugal $(R P=1,05$; IC95\%: 1,01-1,09) e falta de acesso ao serviço de saúde (RP = 0,94; IC95\%: 0,89-0,98) mostraram associação com a diminuição da não utilização de mamografia. Essas associações podem ser parcialmente explicadas pela falta de conhecimento dos riscos, pela inacessibilidade aos serviços de saúde e fatores culturais relacionados ao processo de envelhecimento.

Mamografia; Envelhecimento; Neoplasias da Mama; Programa de Rastreamento

\section{Colaboradores}

C. O. Novaes participou do desenho e concepção do estudo, coleta dos dados, análise e interpretação dos dados e redação do artigo. I. E. Mattos colaborou na concepção do estudo, análise e interpretação dos dados e redação do artigo.

\section{Agradecimentos}

Projeto financiado pelo Conselho Nacional de Desenvolvimento Científico e Tecnológico/Ministério da Saúde (Processo no. 401932/2005-2) e pelo XII Programa de Introdução à Metodologia de Pesquisa em Gênero, Sexualidade e Saúde Reprodutiva - Fundação Ford.

\section{Referências}

1. Lima-Costa MF, Peixoto SV, Giatti L. Tendências de mortalidade entre idosos brasileiros (1980-2000). Epidemiol Serv Saúde 2004; 13:217-28.

2. McCoy CB, Pereyra M, Metsch LR, Collado-Mesa F, Messiah SE, Sears S. A community-based breast cancer screening program for medically underserved women: its effect on disease stage at diagnosis and on hazard of death. Rev Panam Salud Pública 2004; 15:160-7.

3. Lima-Costa MF, Matos DL. Prevalência e fatores associados à realização da mamografia na faixa etária de 50-69 anos: um estudo baseado na Pesquisa Nacional por Amostra de Domicílios (2003). Cad Saúde Pública 2007; 23:1665-73.

4. McPherson CP, Swenson KK, Lee MW. The effects of mammographic detection and comorbidity on the survival of older women with breast cancer. J Am Geriatr Soc 2002; 50:1061-8.

5. Shapiro S. Peridic screening for breast cancer: the HIP Randomized Controlled Trial. Health Insurance Plan. J Natl Cancer Inst Monogr 1997; (22): 27-30.

6. Parvinen I, Helenius H, Pylkkanen L, Anttila A, Immonen-Raiha P, Kauhava L, et al. Service screening mammography reduces breast cancer mortality among elderly women in Turku. J Med Screen 2006; 13:34-40.
7. Fracheboud J, Groenewoud JH, Boer R, Draisma G, Bruijn AE, Verbeek AL, et al. Seventy-five years is an appropriate upper age limit for populationbased mammography screening. Int J Cancer 2006; 118:2020-5.

8. Miller AB. An epidemiological perspective on cancer screening. Clin Biochem 1995; 28:41-8.

9. Amodeo C, Caglià P, Gandolfo L, Veroux M, Brancato G, Immè A. Breast cancer screening in the elderly. Tumori 2003; 89(4 Suppl):173-4.

10. Zappa M, Visioli CB, Ciatto S. Mammography screening in elderly women: efficacy and costeffectiveness. Crit Rev Oncol Hematol 2003; 46: 235-9.

11. U.S. Preventive Services Task Force. Screening for breast cancer: recommendations and rationale. Ann Intern Med 2002; 137(5 Pt 1):344-6.

12. Instituto Nacional de Câncer. Controle do câncer de mama: documento de consenso. Rio de Janeiro: Instituto Nacional de Câncer; 2004.

13. Lairson DR, Chan W, Newmark GR. Determinants of the demand for breast cancer screening among women veterans in the United States. Soc Sci Med 2005; 61:1608-17.

14. Mandelblatt JS, Yabroff KR, Kerner JF. Equitable access to cancer services: a review of barriers to quality care. Cancer 1999; 86:2378-90. 
15. Lane DS, Messina CR. Current perspectives on physician barriers to breast cancer screening. J Am Board Fam Pract 1999; 12:8-15.

16. May DS, Kiefe CI, Funkhouser E, Fouad MN. Compliance with mammography guidelines: physician recommendation and patient adherence. Prev Med 1999; 28:386-94.

17. Fox SA, Siu AL, Stein JA. The importance of physician communication on breast cancer screening of older women. Arch Intern Med 1994; 154:2058-68.

18. Novaes HMD, Braga PE, Schout D. Fatores associados à realização de exames preventivos para câncer nas mulheres brasileiras, PNAD, 2003. Ciênc Saúde Coletiva 2006; 11:1023-35.

19. Reyes-Ortiz CA, Freeman JL, Pelaez M, Markides KS, Goodwin JS. Mammography use among older women of seven Latin American and Caribbean cities. Prev Med 2006; 42:375-80.

20. Instituto Nacional de Câncer. Inquérito domiciliar sobre comportamentos de risco e morbidade referida de doenças e agravos não transmissíveis: Brasil, 15 capitais e Distrito Federal, 2002-2003. Rio de Janeiro: Instituto Nacional de Câncer; 2004.

21. Szwarcwald CL, Leal MC, Gouveia GC, Souza WV. Desigualdades socioeconômicas em saúde no Brasil: resultados de Pesquisa Mundial de Saúde, 2003. Rev Bras Saúde Matern Infant 2005; 5 Suppl 1:S11-22.

22. Lebrão ML, Duarte YAO, organizadores. SABE - Saúde, Bem-Estar e Envelhecimento. O projeto SABE no Município de São Paulo: uma abordagem inicial. Brasília: Organização Pan-Americana da Saúde; 2003.

23. Lima-Costa MF. A saúde dos adultos na Região Metropolitana de Belo Horizonte: um estudo epidemiológico de base populacional. Belo Horizonte: Núcleo de Estudos em Saúde Pública e Envelhecimento, Fundação Oswaldo Cruz/Universidade Federal de Minas Gerais; 2004.

24. Blackman DK, Benet EM, Miller DS. Trends in self reported use mammograms (1989-1997) and $\mathrm{Pa}-$ panicolaou tests (1991-1997): Behavioral Risk Factor Surveillance System. MMWR Surveill Summ 1999; 48:1-22.

25. Breen N, Wagener DK, Brown ML, Davis WW, Ballard-Barbash R. Progress in cancer screening over a decade: results of cancer screening from the 1987, 1992 and 1998 National Health Interview Surveys. J Nat Cancer Inst 2001; 931704-13.

26. Kagay CR, Quale C, Smith-Bindman R. Screening mammography in the American elderly. Am J Prev Med 2006; 31:142-9.

27. Ostbye T, Greenberg GN, Taylor Jr. DH, Lee AM. Screening mammography and Pap tests among older American women 1996-2000: results from the Health and Retirement Study (HRS) and Asset and Health Dynamics Among the Oldest Old (AHEAD). Ann Fam Med 2003; 1:209-17.

28. Sclowitz ML, Menezes AMB, Gigante DP, Tessaro S. Condutas na prevenção secundária do câncer de mama e fatores associados. Rev Saúde Pública $2005 ; 39: 340-9$.
29. Dias-da-Costa JS, Olinto MTA, Bassani D, Marchionatti CRE, Bairros FS, Oliveira MLP, et al. Desigualdades na realização do exame clínico de mama em São Leopoldo, Rio Grande do Sul, Brasil. Cad Saúde Pública 2007; 23:1603-12.

30. NFO Research. AARP/Modern maturity sexuality survey. Washington DC: AARP; 1999.

31. Bluestein D. Preventive services: screening. Health maintenance examinations include screening for diabetes, heart disease, cancer, osteoporosis, hearing and vision loss. Geriatrics 2005; 60:34-9.

32. Tang TS, Solomon LJ, McCracken LM. Cultural barriers to mammography, clinical breast exam, and breast self-exam among Chinese-American women 60 and older. Prev Med 2000; 31:575-83.

33. Edwards N, Jones D. Uptake of breast cancer screening in older women. Age Ageing 2000; 29:131-5.

34. Dias-da-Costa JSD, Olinto MTA, Gigante DP, Menezes AMB, Macedo S, Fuchs SC. Cobertura do exame físico de mama: estudo de base populacional em Pelotas, RS. Rev Bras Epidemiol 2003; 6:39-48.

35. Norman SA, Localio R, Zhou L, Bernstein L, Coates RJ, Flagg EW, et al. Validation of self reported screening mammography histories among women with and without breast cancer. Am J Epidemiol 2003; 158:264-71.

36. Champion VL, Menon U, McQuillen DH, Scott C. Validity of self-reported mammography in low income African American women. Am J Prev Med 1998; 14:111-7.

37. Instituto Brasileiro de Geografia e Estatística. Censo demográfico, 2000. Rio de Janeiro: Instituto Brasileiro de Geografia e Estatística; 2000.

38. Lima-Costa MFF, Guerra HL, Barreto SM, Guimarães RM. Diagnóstico de saúde da população idosa brasileira: um estudo da mortalidade e das internações hospitalares públicas. Inf Epidemiol SUS 2000; 9:23-41.

39. Ramos LR. Fatores determinantes do envelhecimento saudável em idosos residentes em centro urbano: Projeto Epidoso, São Paulo. Cad Saúde Pública 2003; 19:793-7.

40. Loyola Filho AI, Uchoa E, Firmo JOA, Lima-Costa MF. Estudo de base populacional sobre o consumo de medicamentos entre idosos: Projeto Bambuí. Cad Saúde Pública 2005; 21:545-53.

41. Robinson J. Age equality in health and social care. http://ippr.nvisage.uk.com/uploadedFiles/proj ects/Robinson\%20Final\%20Paper.doc (acessado em 03/Abr/2008).

42. Lima-Costa MF, Barreto S, Giatti L, Uchoa E. Desigualdade social e saúde entre idosos brasileiros: um estudo baseado na Pesquisa Nacional por Amostra de Domicílios. Cad Saúde Pública 2003; 19:745-57.

Recebido em 18/Fev/2009

Versão final reapresentada em 10/Jun/2009

Aprovado em 22/Jun/2009 